\title{
Gastric Leiomyosarcoma as a rare cause of gastric outlet obstruction and perforation: a case report
}

\author{
Elroy P Weledji ${ }^{1 *}$, George Enoworock ${ }^{2}$ and Marcelin Ngowe Ngowe ${ }^{1}$
}

\begin{abstract}
Background: Gastrointestinal stromal tumours are the most common mesenchymal malignancies of the gastrointestinal $(\mathrm{Gl})$ tract and gastric leiomyosarcoma represent 1-3\% of gastric malignancies.

Case presentation: We report a case of a 69-year- old black African man who presented with a rare cause of gastric outlet obstruction and duodenal perforation. A Billroth- II gastrectomy was performed and histology confirmed a gastric leiomyosarcoma.

Conclusions: It is important to identify the gastric leiomyosarcoma which is a variant of the more common malignant gastrointestinal stromal tumours as the pathogenesis and management are currently well established. As the facilities for differentiating these are not easily available in resource-limited areas gastrointestinal stromal tumours may remain underdiagnosed and undertreated.
\end{abstract}

Keywords: Gastric outlet obstruction, Leiomyosarcoma, Gastrointestinal stromal tumours

\section{Background}

Gastrointestinal stromal tumours (GISTs) are the most common mesenchymal (non-epithelial) malignancies of the gastrointestinal (GI) tract $[1,2]$. Most arise in the stomach (leiomyosarcoma) representing $0.1-3 \%$ of gastric malignancies, or small intestine, and less frequently in the oesophagus, mesentery, omentum, colon or rectum [1]. Historically, GISTs were considered to be of smooth muscle origin and were generally regarded as benign (leiomyomas) or malignant (leiomyosarcomas). However, only a minority of stromal tumours have the typical features of smooth muscle with some having a more neural appearance and others appearing undifferentiated [3]. The discovery of CD34 expression (80-90\%) and the receptor tyrosine kinase KIT (CD117) in many GISTs suggested that they were a specific entity distinct from smooth muscle tumours [4]. This has led to the widely accepted classification of mesenchymal tumours of the GI tract into GISTS, true smooth muscle tumours, and, far less frequently, true schwann cell tumours [3]. Appropriate management of gastrointestinal mesenchymal tumours requires accurate diagnosis and

\footnotetext{
* Correspondence: elroypat@yahoo.co.uk

'Department of Surgery, Faculty of Health Sciences, University of Buea, PO Box 126 Limbe, S.W. Region, Buea, Cameroon

Full list of author information is available at the end of the article
}

should involve a multidisciplinary approach. Overall, the prognosis of leiomyosarcoma is poor, death often resulting from local spread and/or metastases.

\section{Case presentation}

A 69-yr- old African man was admitted as an emergency with a 2- week history of symptoms of gastric outlet obstruction. He had postpandrial vomiting associated with upper abdominal bloatedness and complained of a constant epigastric pain in the past $48 \mathrm{hrs}$. He had no haemetemesis nor melaena. There was no antecedent history of peptic ulcer disease nor a past history of similar symptoms. He had anorexia, weight loss and constipation. On physical examination he appeared pale and dehydrated. $\mathrm{BP}$ was $100 / 80 \mathrm{mmHg}, \mathrm{HR} 96 / \mathrm{min}$, temperature $36.5^{\circ} \mathrm{C}$. The distension of the upper abdomen was consistent with a dilated stomach. He had no definite signs of peritonism (rebound tenderness, guarding, abdominal rigidity) but mild tenderness on deep palpation of an epigastric mass. The differential diagnosis included a malignant gastric outlet obstruction or an acquired pyloric stenosis from chronic peptic ulcer disease. The haemoglobin level was $10 \mathrm{gm} / \mathrm{l}$, a white cell count was not available but the urea and electrolytes were within normal limits. An erect chest $\mathrm{x}$-ray showed no air under the diaphragm. A plain abdominal $\mathrm{x}$-ray revealed a grossly dilated stomach with food 
contents. An ultrasound scan (U/S) suggested a pyloric tumour. Following resuscitation for $48 \mathrm{hrs}$ with i/v fluids, antibiotics and nasogastric suction, he consented to a laparotomy. At laparotomy there was pus emanating from the left subhepatic space. The stomach was grossly dilated with a 3-4 $\mathrm{cm}$ anterior perforation of the 1st part of the duodenum with surrounding necrosis overlying the common bile duct and gastroduodenal artery. There was seeding of tumour deposits into the serosa and adjacent omentum. The liver appeared normal on palpation. Kocherization of the duodenum allowed a difficult Billroth II partial gastrectomy and a retrocolic gastrojejunostomy fashioned. An open right subhepatic drain was inserted. Intraoperatively he was haemodynamically unstable with hypotension requiring a transfusion of 2 units of blood. Postoperatively, he remained haemodynamically unstable (BP 93/73 $\mathrm{mmHg}$, pulse 97/min,) with associated sweating and oliguria. He was apyrexial but hypoglycaemic with a fasting blood sugar (FBS) of $10.6 \mathrm{~g} / \mathrm{dl}$ (n 14-18 g/dl). He responded to fluid resuscitation and glucose repletion. He tolerated liquid diet on the 5 th post operative day and his haemoglobin level was $12.6 \mathrm{~g} / \mathrm{l}$. On the 7th postoperative day he suddenly became clammy and died. A postmortem was not done. The histology reported a partial gastrectomy specimen measuring $10.0 \times 8.0 \times 7.0 \mathrm{~cm}$, rough surfaced, in folds, brownishyellow colouration and elastic in consistency. Microscopy showed a proliferation of fusiform cells with large, central hyperchromatic nuclei, mottled chromatin and abundant mitosis showing smooth muscle differentiation invading a myxoid and fibro-fatty stroma. This was consistent with a malignant gastrointestinal stroma tumour (malignant GIST) of the leiomyosarcoma type (Figures 1 and 2).

\section{Discussion}

Although almost all perforated gastric ulcers can be effectively managed by laparotomy and omental patch repair, intraoperative biopsy and follow-up endoscopy with repeat biopsy is essential to avoid an underlying malignancy. Large perforation of gastric ulcers usually require distal gastrectomies as in this case [5]. The histology of the resected specimen revealed a malignant GIST (leiomyosarcoma). Gastric leiomyosarcomas represent $10-15 \%$ of mesenchymal tumours and the age distribution and clinical presentation are similar to malignant gastrointestinal stromal tumours (GISTs). The associated systemic symptoms such as fever, night sweats and weight loss are very rare in other sarcomas [1]. The median age at diagnosis is 50-60 years with a slight male predominance. They are often clinically silent until they reach a large size, bleed or rupture [6,7]. Thus, the diagnosis is rarely made preoperatively. Since in many cases the mucosa is normal, a definitive diagnosis for resectable tumour is often made after surgery [7-9]. Seeding of tumour deposits into the serosa

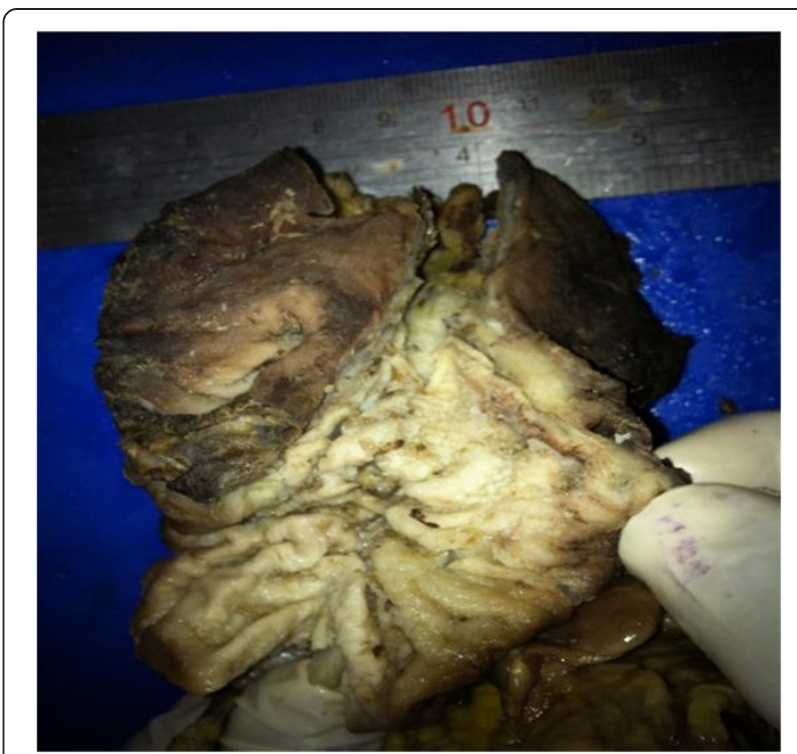

Figure 1 Large gastric leiomyosarcoma $(>8 \mathrm{~cm})$ - high chance of dissemination.

or omentum is almost invariably a sign of malignancy. In this case, the large tumour size greater than $8 \mathrm{~cm}$ and mucosal perforation indicated rapid tumour growth and more likely to be associated with disseminated disease $[1,3]$. The optimisation of this patient's fitness for surgery was compromised by the emergency presentation with gastric outlet obstruction and localized peritonitis. This was exacerbated by the lack of adequate and appropriate postoperative care usually rendered in a high dependency or intensive care unit especially in this patient requiring cardiac and renal support. These are the short-comings of performing major surgery in poor resourced areas. It is known that cancer deaths in hospital involves patient factors, tumour factors and surgeon-related factors [10]. Both tumour (large size

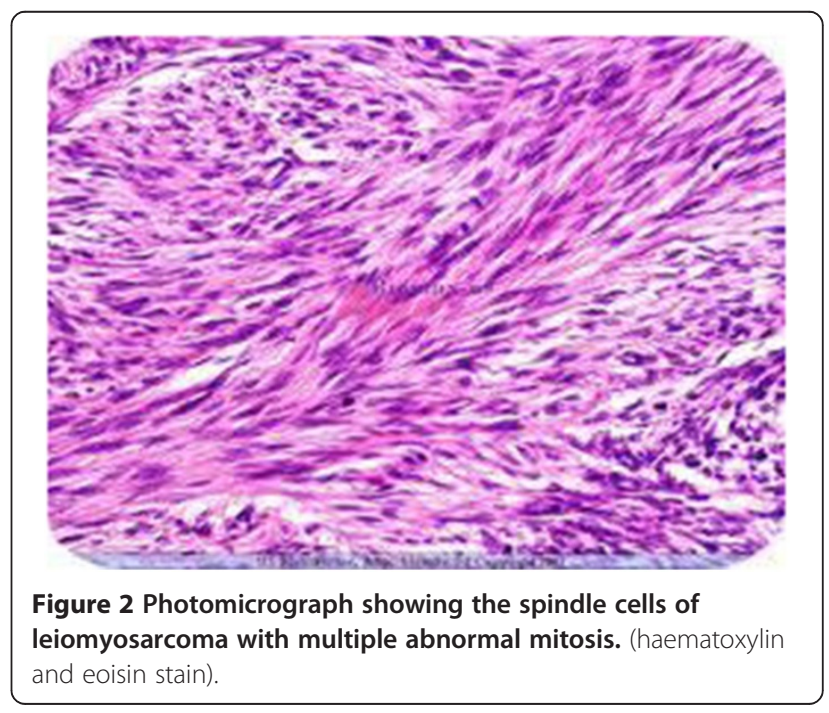


with obstruction and perforation) and patient factors were not favourable. The probable immediate cause of death was cardiac failure as he remained cardiovascularly unstable perioperatively.

In resourced areas, initial diagnosis of malignant GIST is based on imaging. Endoscopic ultrasonography (EUS) especially of the oesophagus, stomach, duodenum and anorectum can confirm the diagnosis of small lesions less than $2 \mathrm{~cm}$. For large tumours, computed tomography (CT) of chest, abdomen and pelvis would assess primary tumour extension and stage for metastases. Percutaneous (US or CT -guided) or laparoscopically- guided biopsies are not used in resectable disease due to the risk of tumour rupture or seeding unless it may result in a change of treatment [8]. Laparoscopy may be considered to stage for peritoneal and distant spread of disease $[7,9]$. For patients with unresectable and/or metastatic tumours, an endoscopic or percutaneous biopsy is taken for a definitive diagnosis before treatment.

As the morphological spectrum of GISTs is wider than previously recognized, macroscopic examination of the site of the resected tumour, with an adequate sampling for histological examination and for immunohistochemistry should be performed [9]. In this case, the histopathological diagnosis of a malignant GIST would have been supported by a positive expression of CD34 or CD117 [4,11]. Evaluation of respectability of gastrointestinal stromal tumour is determined by the surgeon and depends on the stage and the individual patient's fitness for surgery $[7,8]$. As they rarely metastasise to lymph nodes, extended lymphadenectomy is seldom warranted. En bloc resection of involved adjacent organs is necessary for oncological clearance $[8,9]$. As metastasis is primarily haematogenous, the five year survival following surgical (RO) resection is 37$54 \%$ [5]. Neither palliative or adjuvant radiotherapy nor standard chemotherapy has been shown to be of benefit $[7,8]$. The early results suggest that molecular therapy with the tyrosine kinase inhibitor, imitanib mesylate, may play an important role as adjuvant therapy following GIST resection. It increases recurrence -free survival as shown by contrast enhanced CT scanning $[12,13]$.

\section{Conclusion}

It is important to identify the gastric leiomyosarcoma which is a variant of the more common malignant gastrointestinal stromal tumour (GISTs) whose pathogenesis and management are currently well established. As the facilities for differentiating these are not easily available gastrointestinal stromal tumours may remain underdiagnosed and undertreated in resource-limited areas.

\section{Consent}

"Written informed consent was obtained from the next of kin to the deceased for the case report and accompanying images to be published. A copy of the written consent is available for review by the editor-in- chief of this journal".

\section{Competing interests}

The authors declare that they have no competing interests.

\section{Authors' contributions}

EPW was the surgeon and carried out the design of the case report and drafted the manuscript; GE was the pathologist who provided the histology report of the resected specimen and participated in the article's design; MNN participated in the design and coordination. All authors read and approved the final manuscript.

\section{Acknowledgements}

We thank the histopathology department for processing the histology on the resected gastric specimen.

\section{Author details}

${ }^{1}$ Department of Surgery, Faculty of Health Sciences, University of Buea, PO Box 126 Limbe, S.W. Region, Buea, Cameroon. 'Department of Pathology, Faculty of Health Sciences, University of Buea, Buea, Cameroon.

Received: 29 March 2014 Accepted: 25 July 2014

Published: 29 July 2014

References

1. Appelman HD: Smooth muscle tumours of the gastrointestinal tract. What we know now that Stout didn't know. Am J Surg Pathol 1986, 10(S1):83-99.

2. Lehnert T, Sinn HP, Waldherr R: Surgical treatment of soft tissue tumours of the stomach. Eur J Surg Oncol 1990, 10:352-359.

3. Graadt van Roggen JF, van Velthuysen MLF, Hogendoorn PCW: The histopathological differential diagnosis of gastrointestinal stromal tumours. J Clin Pathol 2001, 54:96-102.

4. Van de Rijn M, Hendrickson MR, Rouse RV: The CD34 expression by the Gastrointestinal stromal tumours. Hum Pathol 1994, 25:661-771.

5. Leeman MF, Skouras C, Paterson Brown S: The management of perforated gastric ulcers. Int J Surg 2013, 11(4):322-324

6. Shepherd JA: Perforation of leiomyosarcoma of stomach. Br J Surg 1950, 97:479-481.

7. Grant CS, Kim CH, Farrugia G, Zinsmeister A, Goellner JR: Gastric Leiomyosarcoma. Prognostic factors and surgical management. Arch Surg 1991, 126:985-990.

8. O'Hanlon DM, Griffin SM: Management of other oesophageal and gastric neoplasms. In Upper Gastrointestinal surgery. A companion to specialist surgical practice. Edited by Michael Griffin S, Raimes SA. London, NW1 7DX, England: WB Saunders Company Ltd; 2000.

9. Connolly EM, Gaffney E, Reynolds JV: Gastrointestinal stromal tumours. $\mathrm{Br} J$ Surg 2003, 90:1178-1186.

10. Fielding LP, Phillips RK, Fry JS, Hittinger R: Prediction of outcome after curative surgery for large bowel cancer. Lancet 1986, ii:904-906.

11. Fletcher JA: The molecular pathogenesis of gastrointestinal stromal tumours. Monographs in Gastrointestinal stromal tumors 2003, 1(1\&2):15-20.

12. Dematteo RP, Ballman KV, Antonescu CR, Maki RG, Pisters PW, Demetri GD, Blackstein ME, Blanke CD, von Mehren M, Brennan MF, Patel S, McCarter MD, Polikoff JA, Tan BR, Owzar K, American College of Surgeons Oncology Group (ACOSOG) Intergroup Adjuvant GIST Study Team: Adjuvant imatinib mesylate after resection of localized, primary gastrointestinal stromal tumour: a randomized, double-blind, placebo-controlled trial. Lancet 2009, 373:1097-1104.

13. Kantharia CV, Irpatigire R, Prabbhu RY, Bapat RD, Supe AN: Varied presentations of gastrointestinal stromal tumour. Indian J Surg 2008 70:318-321.

\section{doi:10.1186/1756-0500-7-479}

Cite this article as: Weledji et al:: Gastric Leiomyosarcoma as a rare cause of gastric outlet obstruction and perforation: a case report. BMC Research Notes 2014 7:479. 